\title{
Breaking down BRCA1
}

Two months ago, this journal added its voice to the chorus of approval that accompanied the isolation of a gene for hereditary breast and ovarian cancer - BRCA1. This long-awaited event, orchestrated by Mark Skolnick of the University of Utah and Myriad Genetics, hinged on the discovery of five mutations in a large gene in affected families ${ }^{1}$, and was supported by the presence of four germline mutations (including one of the family mutations) in a survey of 44
- Two missense mutations replace the last pair of cysteine residues in the $\mathrm{Cys}_{3}-\mathrm{His}-\mathrm{Cys}_{4}$ zinc finger domain of BRCA1. These substitutions Cys61Gly (seen in two probably unrelated families by King's group ${ }^{5}$ and Cys64Gly (Weber ${ }^{3}$ ) support the physiological significance of this motif and the putative role of BRCAl as a transcription factor. By contrast, a frameshift mutation removes just the last 11 our of 1,863 residues yet leads to early-onset breast cancer ${ }^{5}$.

- About $70 \%$ of the mutations result in the loss or premature termination of protein synthesis, many stemming from small insertions or deletions. This seems to support the 'two-hit' hypothesis, especially given further evidence that it is always the wild-type $B R C A 1$ allele that is lost in tumours ${ }^{5}$. BRCA1 bears the hallmarks of a tumour suppressor gene.

- A handful of mutations recur, in addition to Cys61Gly (see above) and the original Met1775Arg substitution ${ }^{1,2}$. Narod and colleagues describe two frameshift mutations at opposite ends of the gene-

putative sporadic cancer patients ${ }^{2}$. The evidence was convincing; nevertheless the final title of the cloning paper ${ }^{1}$ was toned down slightly from its original draft:Skolnick's team announced 'a strong candidate' for BRCA1.

With the flurry of publicity surrounding the discovery long before Science could publish the reports, rival researchers anxious to begin their own family studies had to wait three long weeks, until the day of publication, for the complete nucleotide sequence of $B R C A 1$ to be unveiled in GenBank. Within days, oligonucleotide primers were prepared to start the genetic analysis. Less than a week later, some groups had already found sequence changes in their own patient samples.

The results of some of this frenetic activity are to be found in this issue, in the form of a triad of reports ${ }^{3-5}$ from the groups of Barbara Weber (University of Pennsylvania) and Francis Collins (NIH); Steven Narod (McGill University) in conjunction with Skolnick; and Mary-Claire King's group at Berkeley. Between them, these researchers describe a total of 31 mutations in $B R C A 1$ from various families, of which 22 are distinct. A few interesting points stand out: 185delAG and 5382insC (also reported in one of the original Utah families ${ }^{1}$ ) — in affected members of four separate Canadian families. Several other mutations appear in more than one family but overall, hopes of finding a 'common' mutation appear bleak.

- Families with confirmed mutations in $B R C A 1$ seem to have a lower age of onset than those without, and a greater likelihood of multiple ovarian cancers ${ }^{3,4}$. The 5382insC mutation may be associated with a wide range of different cancers ${ }^{4}$, but there is no obvious relationship between mutation position and phenotype ${ }^{5}$.

- Prospects for wide-scale screening of $B R C A 1$ look dim. A total of 100 breast and breast/ovarian cancer families were screened ${ }^{3-5}$, but mutations were detected in just 31. Many possible explanations exist, including the genetic heterogeneity of hereditary breast cancer; insensitivity of mutation detection methods; and failure to screen the entire $B R C A 1$ gene.

1. Miki ,Y. et al. Science 286, 66-71 (1994).

2. Futreal, P.A. et al. Science 286, 120-122 (1994).

3. Castilla, L.H. et al. Nature Genet. 8, 387-391 (1994).

4. Simard, J. et al. Neture Genet. 8, 392-398 (1994).

5. Friedman, L.S. et al. Nature Genet. 8, 399-404 (1994). 